\title{
Highly sensitive Raman spectroscopy with low laser power for fast in-line reaction and multiphase flow monitoring
}

Frank Braun, ${ }^{\dagger,} \perp$ Sebastian Schwolow, ${ }^{\dagger} \perp$ Julia Seltenreich, ${ }^{\dagger}$ Norbert Kockmann, ${ }^{\S}$ Thorsten Röder, ${ }^{\ddagger}$ Norbert Gretz," Matthias Rädle ${ }^{\dagger, *}$

${ }^{\dagger}$ Mannheim University of Applied Sciences, Institute of Process Control and Innovative

Energy Conversion, Paul-Wittsack-Str. 10, 68163 Mannheim, Germany

${ }^{\ddagger}$ Mannheim University of Applied Sciences, Institute of Chemical Process Engineering, PaulWittsack-Str. 10, 68163 Mannheim, Germany

${ }^{\S}$ TU Dortmund University, Biochemical and Chemical Engineering, Equipment Design, EmilFigge-Straße 68, 44227 Dortmund, Germany

"Medical Research Center, Medical Faculty Mannheim, University of Heidelberg, TheodorKutzer-Ufer 1-3, 68167 Mannheim, Germany

${ }^{\perp}$ F.B. and S.S. contributed equally to the experimental work.

*Corresponding Author. E-mail: m.raedle@hs-mannheim.de. Telephone: +49621 761-508-11 


\section{A. Influence of the fiber diameter on signal intensity and spectral resolution}

In low energy experiments fiber diameter and integration times were varied to investigate the influence of the parameters on the signal intensity and on the spectral resolution. The Raman signal of cyclohexane in a quartz cuvette was measured with the focusing probe and an excitation intensity of $10 \mathrm{~mW}$. Fig. S1a shows the increase of the signal intensity (peak height) with increasing fiber cross section. The loss of spectral resolution is represented by the increasing full width at half maximum of the Raman peak at $1446 \mathrm{~cm}^{-1}$ (Fig. S1b). The use of larger fiber diameters can be necessary to increase the signal-to-noise ratio if short integration times are required (Fig. S2a, $20 \mathrm{~ms}$ ). With longer integration times, smaller fiber diameters can be used to obtain a better spectral resolution (Fig. S2b, $500 \mathrm{~ms}$ ).
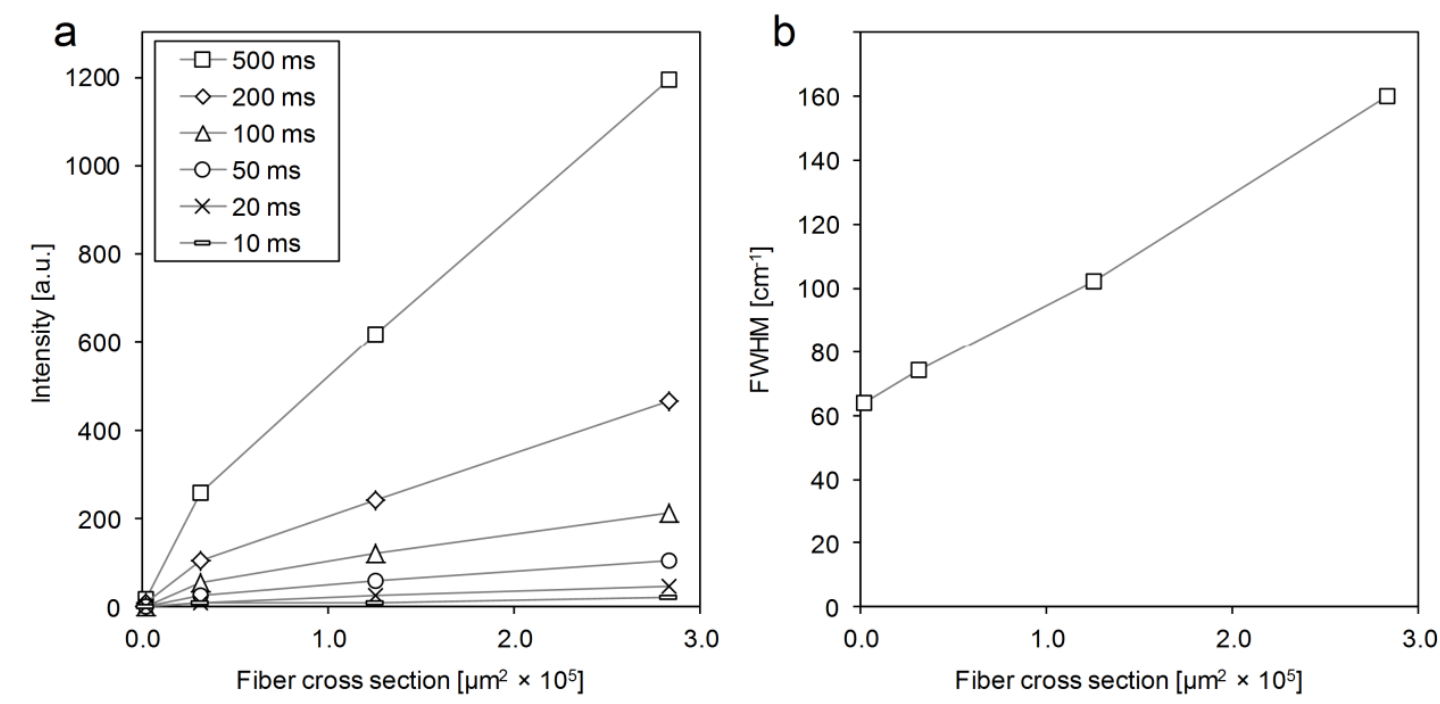

Figure S1. (a) Difference of Raman intensities between 1446 and $1664 \mathrm{~cm}^{-1}$ for different integration times as a function of the fiber cross section $(50,200,400$, and $600 \mu \mathrm{m}$ fibers) determined at low intensity experiments with $10 \mathrm{~mW}$ excitation intensity. (b) Full width at half maximum of the $1446 \mathrm{~cm}^{-1}$ Raman peak as a function of the fiber cross section. 

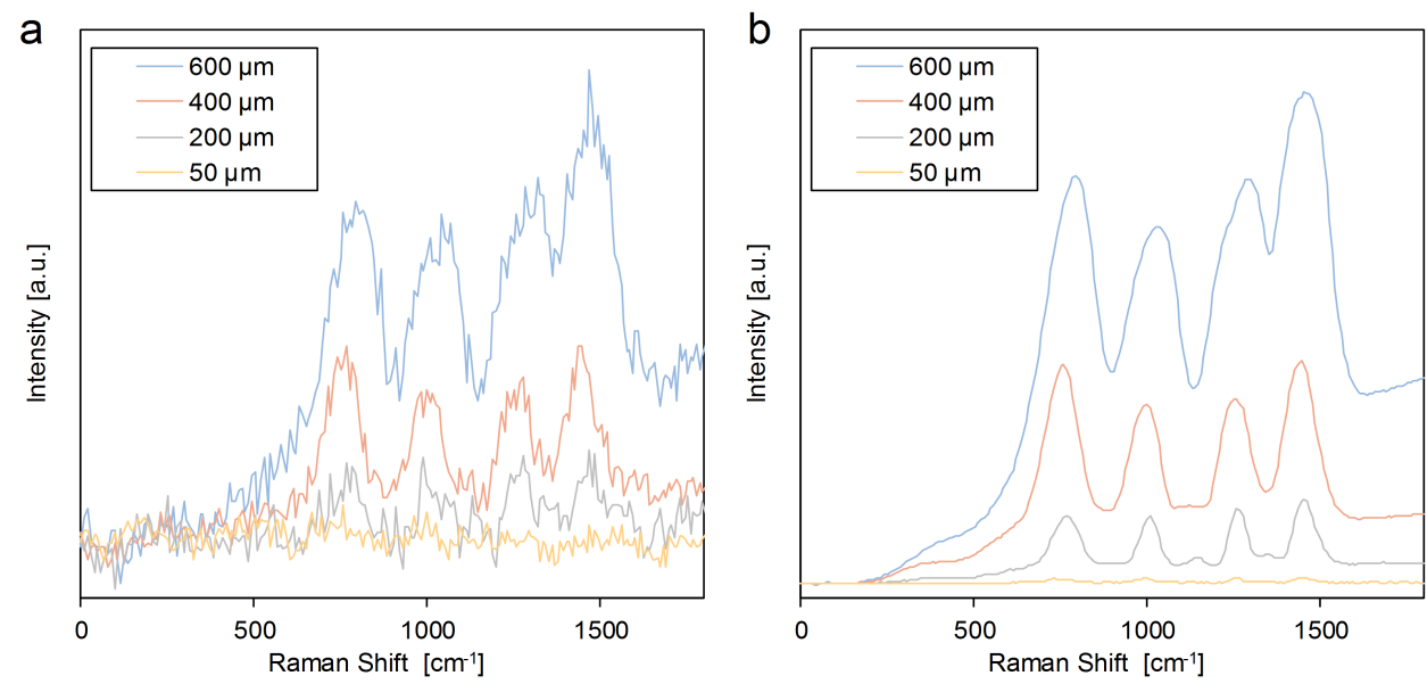

Figure S2. Single Raman spectra from low intensity experiments with $10 \mathrm{~mW}$ excitation intensity with different fiber diameters and integration times of $20 \mathrm{~ms}$ (a) and $500 \mathrm{~ms}$ (b).

A direct comparison between low and high energy measurements is given in Fig. S3. The dashed line represents the signal intensity of a Raman measurement under typical experimental conditions with $100 \mathrm{~mW}$ excitation intensity and a small entrance cross section (50 $\mu \mathrm{m}$ fiber). For comparison, the $10 \mathrm{~mW}$ curves are plotted and allow for estimating the required increase of fiber diameter or integration time to obtain similar signal intensity. The change from $50 \mu \mathrm{m}$ to a $400 \mu \mathrm{m}$ fiber diameter compensates for a laser power reduction by the factor of 10 . 


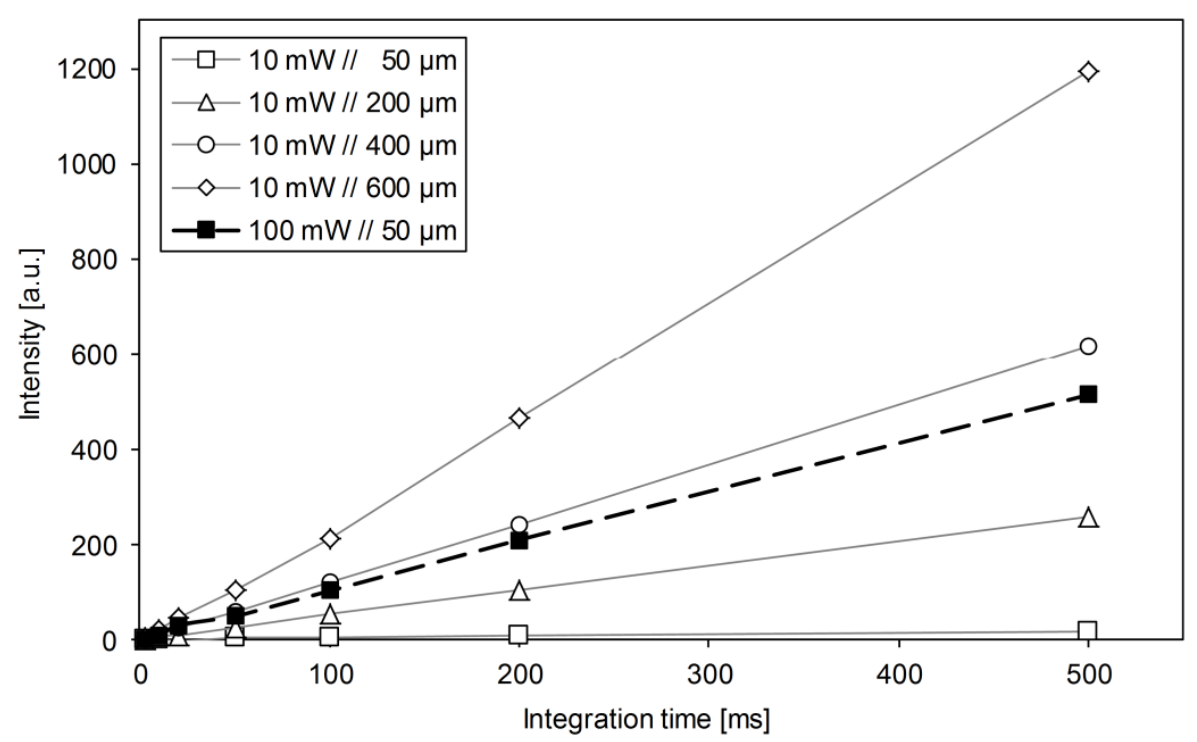

Figure S3. Difference of Raman intensities between 1446 and $1664 \mathrm{~cm}^{-1}$ as a function of the integration time. The results of low intensity experiments with varying fiber cross section (50, 200, 400, and $600 \mu \mathrm{m}$ fibers) are compared to a high intensity experiment with the smallest fiber diameter (50 $\mu \mathrm{m}$ fiber, $100 \mathrm{~mW}$ excitation intensity).

\section{$\underline{\text { B. Minimum limit of detection for ethyl acrylate }}$}

The minimum detection limit of ethyl acrylate with unattenuated laser intensity $(300 \mathrm{~mW})$ was determined to investigate the capabilities of the system at low concentrations. Ethyl acrylate was diluted in water and measured through the wall of a glass vial with the coaxial probe and a $400 \mu \mathrm{m}$ fiber. Due to the low concentrations, the generally weak Raman signal of water cannot be neglected. The water peak at $1640 \mathrm{~cm}^{-1}$ (Pouchert, 1981) adds to the ethyl acrylate peak between $1559 \mathrm{~cm}^{-1}$ and $1934 \mathrm{~cm}^{-1}$ (Fig. S4). Therefore, the ethyl acrylate signal was calculated from the mean intensity of the peak after baseline correction and subtraction of the water signal. Noise was determined as the root-mean-square error for a parabolic fit in the spectral range between $1950 \mathrm{~cm}^{-1}$ and $2060 \mathrm{~cm}^{-1}$. As a result of a linear fit for the SNR/ 
concentration plot (Fig. S4), a noise equivalent concentration of $0.8 \mathrm{mM}$ can be estimated for ethyl acrylate in water.
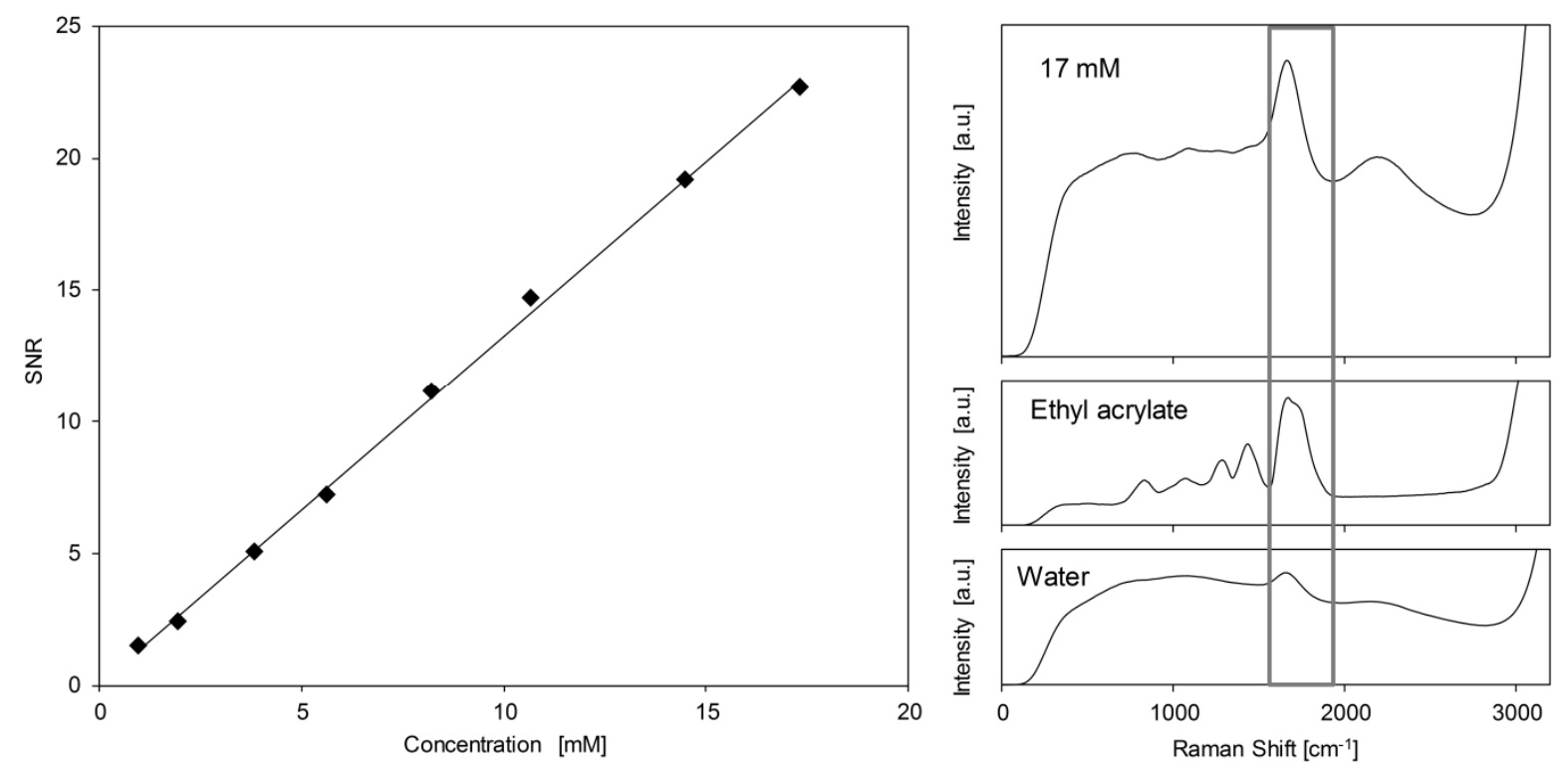

Figure S4. Signal-to-noise ratios (SNR) calculated from Raman spectra of ethyl acrylate in water and determined at different concentrations from 20 spectra with $4 \mathrm{~s}$ acquisition time each.

\section{REFERENCES}

Pouchert, C. J. The Aldrich library of infrared spectra; Sigma Aldrich: Milwankee, 1981. 